\title{
EFEITOS DO ULTRA-SOM DE BAIXA INTENSIDADE NA VEIA AURICULAR DE COELHOS ${ }^{1}$
}

\author{
Marcelo Araújo $0^{2}$ \\ José Carlos Costa Baptista-Silva ${ }^{3}$ \\ Paulo de Oliveira Gomes ${ }^{4}$ \\ Humberto de Oliveira Campos ${ }^{5}$ \\ Neil Ferreira Novo ${ }^{6}$ \\ Yara Juliano
}

\begin{abstract}
Araújo M, Baptista-Silva JCC, Gomes PO, Novo NF, Juliano Y. Efeitos do ultra-som de baixa intensidade na veia auricular de coelhos. Acta Cir Bras [serial online] 2003 Jan-Fev;18(1). Disponível em URL: http://www.scielo.br/acb.
\end{abstract}

RESUMO - Objetivo: Estudar a ação do ultra-som na veia auricular de coelhos. Métodos: Vinte coelhos foram divididos em dois grupos de dez animais diferindo com relação ao local da aplicação, do ultra-som, o modo e o intervalo de tempo para a análise histopatológica (3 e 7 dias). Os animais foram submetidos à aplicação de ultra-som contínuo e pulsado em dois segmentos venosos da orelha previamente determinados. Cada animal foi o seu próprio controle. Empregou-se a freqüência de $3 \mathrm{MHz}$, intensidade de $3 \mathrm{~W} / \mathrm{cm}^{2}$ nos ciclos pulsado e contínuo por 10 minutos, de forma estacionária. O grupo I foi submetido a eutanásia após 3 dias e o grupo II em 7 dias contemplando a fase aguda do processo inflamatório. Empregou-se o teste exato de Fisher e o teste de Mc Nemar para análise estatística. Resultados: Obteve-se trombose venosa e aumento de linfócitos de forma significativa $(p=0,032)$ nos grupos tratados com o modo contínuo. $\mathrm{O}$ modo pulsado não provocou efeitos deletérios. Outros achados foram congestão, edema, hemorragia e lesão da parede vascular. Conclusões: O ultra-som pulsado não provoca qualquer alteração na parede vascular nas condições do experimento. $\mathrm{O}$ ultra-som contínuo induz a trombose venosa e aumento dos linfócitos de forma significativa.

DESCRITORES - Ultra-som. Terapia por ultra-som. Vascular. Veias. Coelho.

\section{INTRODUÇÃO}

O ultra-som é uma importante forma de energia capaz de produzir efeitos biológicos no organismo quando utilizado em potência maior que $100 \mathrm{~mW} / \mathrm{cm}^{2}$, considerado o limite superior para os sistemas ultrasônicos destinados ao diagnóstico. Vários procedimentos terapêuticos utilizam a energia ultra-sônica de diferentes maneiras. As potências utilizadas podem ser tão baixas como 1 a $5 \mathrm{~W} / \mathrm{cm}^{2}$, empregadas correntemente

1. Este trabalho é parte da Tese apresentada à Universidade Federal de São Paulo - Escola Paulista de Medicina, para a obtenção do Título de Mestre em Técnica Operatória e Cirurgia Experimental. Experimento realizado no laboratório de anatomia veterinária da Universidade Estadual de Santa Cruz. Ilhéus - BA

2. Mestre em Técnica Operatória e Cirurgia Experimental pela Escola Paulista de Medicina - Universidade Federal de São Paulo (UNIFESP-EPM). Angiologista e Cirurgião Vascular Chefe do Departamento de Cirurgia da Santa Casa de Itabuna - BA.

3. Professor Adjunto Livre-Docente da Disciplina de Cirurgia Vascular do Departamento de Cirurgia da UNIFESP-EPM.

4. Professor Doutor Adjunto do Programa de Pós-graduação em Técnica Operatória e Cirurgia Experimental da UNIFESP-EPM.

5. Professor Doutor Assistente do Curso de graduação em Técnica Operatória e Cirurgia Experimental da Escola Bahiana de Medicina

6. Professor do Departamento de Medicina Preventiva e Bioestatística do curso de Pós-graduação em Técnica Operatória e Cirurgia Experimental da Universidade Federal de São Paulo - Escola Paulista de Medicina.

7. Professora do Departamento de Medicina Preventiva e Bioestatística do curso de Pós-graduação em Técnica Operatória e Cirurgia Experimental da Universidade Federal de São Paulo - Escola Paulista de Medicina 
em fisioterapia, alcançando até níveis acima de 3000 $\mathrm{W} / \mathrm{cm}^{2}$ utilizado em litotripsia extracorpórea ${ }^{1}$. Os efeitos são de duas categorias $2,3,4$ : térmicos e não térmicos. Entretanto existe o risco real de ocorrerem sérios danos biológicos dependendo da intensidade, da freqüência e do sistema orgânico em questão. O sistema circulatório tem a peculiaridade única de interagir com o ultra-som, pois apresenta partículas em movimento e vasomotricidade baseada num complexo controle neuro-humoral, o que modifica a dissipação e absorção do feixe sonoro. Respostas como alteração da coagulação 5, 6,7 e fibrinólise ${ }^{8}$, vasomotricidade ${ }^{9}$, estímulo angiogênico e outros já têm sido descritos em situações específicas e controladas de emprego do ultra-som. É fato que esta forma de energia é capaz de alcançar grandes profundidades, entretanto sabe-se que estruturas nobres e delicadas encontram-se no trajeto do feixe ultra-sônico estando sujeitas, portanto à sua ação. O sistema venoso, devido a sua posição superficial, em alguns locais imediatamente sob a pele, encontra-se evidentemente mais exposto às agressões externas. Poucas referências foram encontradas na literatura sobre estudos a este respeito ${ }^{10,11,12,13,14}$.

Com o objetivo de avaliar o risco potencial da utilização do ultra-som de baixa intensidade no sistema venoso, o que corresponde à faixa de potência utilizada em fisioterapia, procedeu-se este estudo experimental em coelhos.

\section{MÉTODOS}

O experimento foi aprovado pela Comissão de Ética em Pesquisa da Universidade Federal de São Paulo - Escola Paulista de Medicina baseando-se na legislação pertinente em vigor.

Utilizou-se vinte coelhos (Oryctolagus cuniculus) da linhagem Nova Zelândia, brancos, machos jovens pesando entre 2 e $3 \mathrm{~kg}$ procedentes da Universidade
Estadual de Santa Cruz (UESC), Ilhéus-Bahia. Os animais foram mantidos na instituição por dez dias, sendo três para aclimatação e sete para o experimento. Optou-se pelo sistema de manejo fechado. A experimentação foi realizada no laboratório de anatomia veterinária da UESC, com sistema de ventilação exaustão forçada ${ }_{2}$ períodos de luminescência natural, temperatura média de $20^{\circ} \mathrm{C}$, ruído mínimo e umidade em torno de $50 \%$. A dieta foi livre até o momento do experimento sendo constituída de água e ração comercial granulada ${ }^{1}$ ad libtum. Cada animal foi mantido em gaiolas de $1 \mathrm{~m}^{2}$ dispostas em fileiras sobre as bancadas de aço inoxidável, de maneira que recebeu a mesma intensidade de luz, circulação de ar e temperatura.

A escolha dos animais foi feita ao acaso por sorteio. Constituíram-se dois grupos com dez animais cada, que foram identificados por números de 1-10 para o grupo I e 11-20 para o grupo II. A identificação foi feita com caneta de tinta preta indelével nas bases das orelhas fora da área do trajeto vascular.

Cada animal serviu como seu próprio controle. As áreas a serem tratadas foram previamente definidas da seguinte forma: considerando-se uma certa homogeneidade no diâmetro da veia auricular lateral (vena auricularis lateralis), foram enumerados quatro segmentos para serem utilizados como áreas tratadas e controles que por sua vez originaram oito subgrupos (Quadro 1).

Segmento A - porção proximal da veia auricular lateral da orelha direita;

Segmento B - porção distal da veia auricular lateral da orelha direita;

Segmento C - porção distal da veia auricular lateral da orelha esquerda;

Segmento D - porção proximal da via auricular lateral da orelha esquerda.

Em ambos os grupos foram demarcados segmentos venosos que constituíram os seguintes subgrupos:

QUADRO 1 - Subgrupos quanto aos segmentos venosos tratados e controles.

\begin{tabular}{c|l|c|c}
\hline IA & Segmento proximal da orelha direita & Sham & 3 dias \\
\hline IB & Segmento distal da orelha direita & US contínuo & 3 dias \\
\hline IC & Segmento distal da orelha esquerda & Sham & 3 dias \\
\hline ID & Segmento proximal da orelha esquerda & US pulsado & 3 dias \\
\hline \multicolumn{5}{|l|}{} & Sham & 7 dias \\
\hline IIA & Segmento proximal da orelha direita & US pulsado & 7 dias \\
\hline IIB & Segmento distal da orelha direita & Sham & 7 dias \\
\hline IIC & Segmento distal da orelha esquerda & US contínuo & 7 dias \\
\hline IID & Segmento proximal da orelha esquerda &
\end{tabular}

1 DU Coelho. Anhangüera, SãoPaulo-SP 
Os animais foram imobilizados manualmente para a remoção cuidadosa dos pêlos com lâmina de barbear na área de interesse.

Utilizou-se o anestésico de uso veterinário xilazina ${ }^{2}$, na dose de $2 \mathrm{mg} / \mathrm{kg}$ de peso por via intramuscular profunda no membro posterior direito. Após a sedação e controle do animal colocou-se em decúbito lateral com a orelha apoiada sobre uma lâmina de borracha sintética e isopor para minimizar a reflexão do ultrasom.

Utilizou-se um equipamento gerador de ultra-som disponível comercialmente ${ }^{3}$ com ciclos contínuo e pulsado.

Manteve-se a calibração original de fábrica do equipamento. A potência foi de $3 \mathrm{~W} / \mathrm{cm}^{2}$, com $3 \mathrm{MHz}$ de freqüência durante dez minutos nos modos contínuo e pulsado. O transdutor foi mantido em posição por um suporte universal de laboratório.

Aplicou-se gel sobre o trajeto venoso e o transdutor foi acoplado a um redutor de superfície com $1 \mathrm{~cm}^{2}$ de área. Entre o transdutor e o redutor de superfície também se utilizou gel a fim de facilitar a passagem do ultra-som. A forma de aplicação foi estacionária. A distância do transdutor à pele foi estabelecida em 0,5 centímetro.

O estudo estatístico foi feito pelo serviço de bioestatística do Departamento de Medicina Preventiva e Bioestatística da Universidade Federal de São Paulo Escola Paulista de Medicina (UNIFESP - EPM).

Para análise dos resultados foram aplicados os seguintes testes:

1. Teste exato de Fisher $^{15}$ para comparar os grupos I e II em relação à ocorrência das variáveis estudadas em cada procedimento.

2. Teste de Mc Nemar ${ }^{16}$ para estudar para cada variável as discordâncias entre os dois procedimentos.

Fixou-se em 0,05 ou $5 \%$ (alfa £ 0,05 ) o nível de rejeição da hipótese de nulidade assinalando-se com um asterisco os valores significantes.

\section{RESULTADOS}

Lesões de natureza inflamatória como congestão da luz venosa e edema perivascular (Figura 1), e outras mais graves como hemorragia, trombose (Figura 2) e até destruição total da parede vascular (Figura 3 ) foram encontradas.

2 Coopazine, Laboratório Mallinckrodt Vet. Ltda., Cotia SP. 3 KROMAN TRIGHER - MEGSON KC-709.

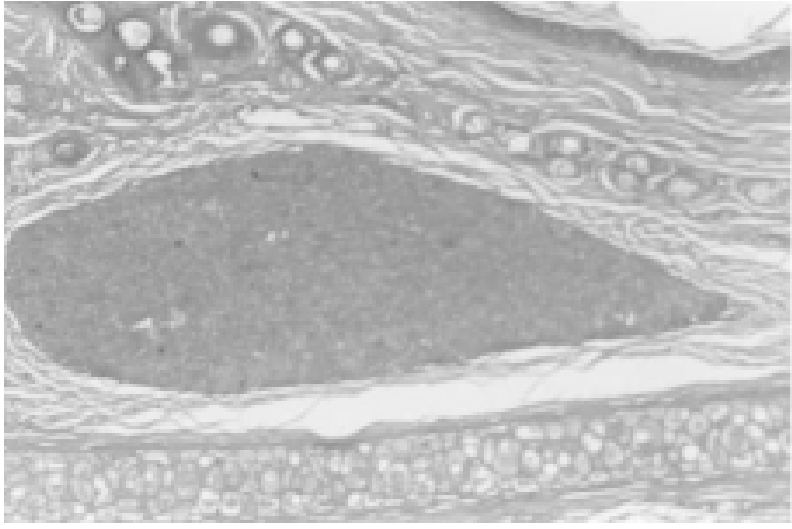

FIGURA 1 - Fotomicrografia mostrando congestão venosa e edema perivascular.

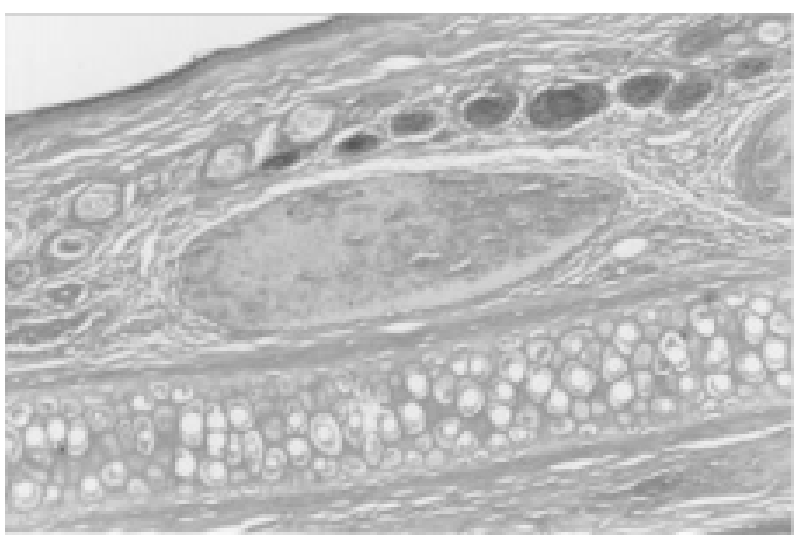

FIGURA 2 - Fotomicrografia mostrando trombo venoso organizado e aderido à parede vascular.

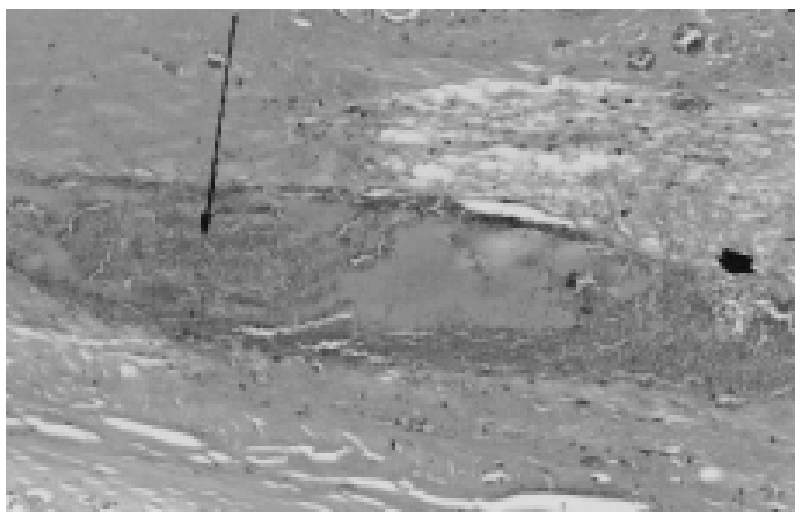

FIGURA 3 - Fotomicrografia mostrando destruição da parede vascular (seta larga) e organização do trombo (seta estreita).

Os achados histopatológicos do grupo II revelaram alterações significantes das estruturas vasculares normais quando submetidas ao ultra-som contínuo. A trombose ocorreu em $44,44 \%$ dos casos tratados com esta modalidade de ultra-som verificado no subgrupo II D $(p=0,032)$. A ocorrência da congestão e edema 
não chegou a ser estatisticamente significante, embora a utilização do ultra-som pulsado tenha promovido um aumento considerável do edema no subgrupo I D, sendo a única observação digna de nota com a utilização desta modalidade de ultra-som. A presença de linfócitos ocorreu de forma significante $(\mathrm{p}=0,032)$ no subgrupo II D (7 dias), marcando o final da fase aguda (Figura 4, Tabela 1).

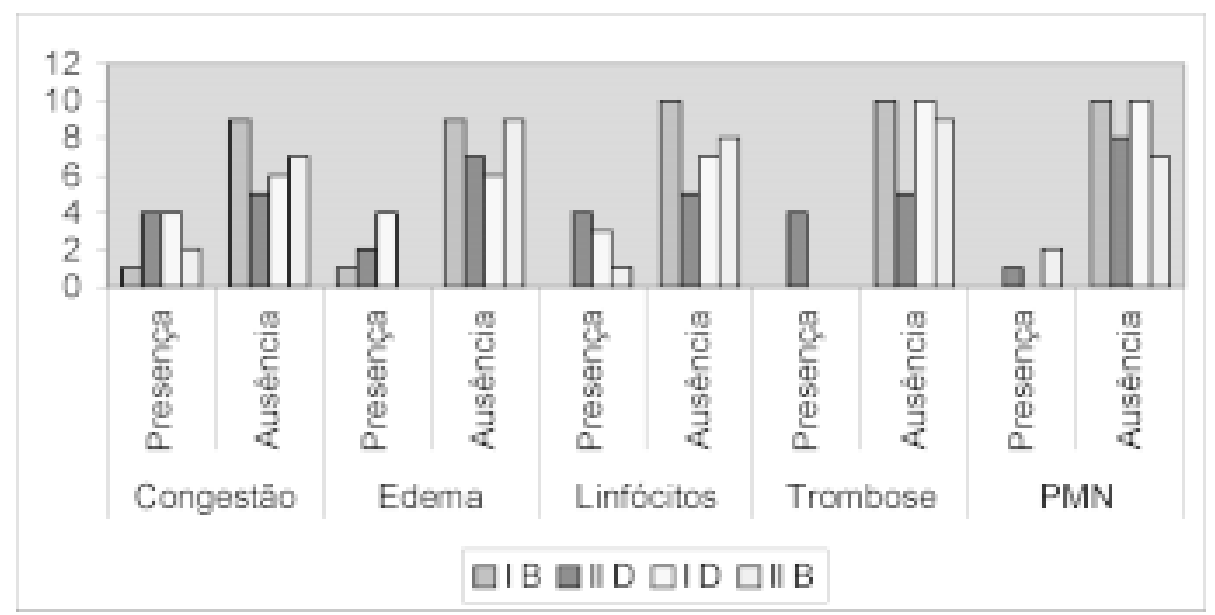

FIGURA 4 - Ocorrência das alterações histopatológicas em veias expostas ao ultra-som nos modos contínuo e pulsado $3 \mathrm{~W} / \mathrm{cm}^{2}$ e $3 \mathrm{MHz}$.

TABELA 1 - Alterações histológicas nas veias das orelhas de coelhos expostas ao ultra-som a $3 \mathrm{Wcm}^{2}, 3 \mathrm{MHz}$ nos modos contínuo e pulsado por 10 minutos.

\begin{tabular}{|c|c|c|c|c|c|c|}
\hline VARIÁVEL & GRUPO & MODO & PRESENÇA & AUSÊNCIA & $\begin{array}{c}\text { PRESENÇA } \\
\% \\
\end{array}$ & $\begin{array}{c}\text { TESTE DE } \\
\text { FISHER }\end{array}$ \\
\hline \multirow[t]{4}{*}{ Congestão } & I B & Contínuo & 1 & 9 & $10,00 \%$ & \\
\hline & II D & Contínuo & 4 & 5 & $44,44 \%$ & $\mathrm{P}=0,119$ \\
\hline & I D & Pulsado & 4 & 6 & $40,00 \%$ & \\
\hline & II B & Pulsado & 2 & 7 & $22,22 \%$ & $\mathrm{P}=0,369$ \\
\hline \multirow[t]{4}{*}{ Edema } & I B & Contínuo & 1 & 9 & $10,00 \%$ & \\
\hline & II D & Contínuo & 2 & 7 & $22,22 \%$ & $\mathrm{P}=0,458$ \\
\hline & I D & Pulsado & 4 & 6 & $40,00 \%$ & \\
\hline & II B & Pulsado & 0 & 9 & $0,00 \%$ & $\mathrm{P}=0,054$ \\
\hline \multirow[t]{4}{*}{ Linfócitos } & I B & Contínuo & 0 & 10 & $0,00 \%$ & \\
\hline & II D & Contínuo & 4 & 5 & $44,44 \%$ & $\mathrm{P}=\mathbf{0 , 0 3 2 *}$ \\
\hline & I D & Pulsado & 3 & 7 & $30,00 \%$ & \\
\hline & II B & Pulsado & 1 & 8 & $11,11 \%$ & $\mathrm{P}=0,332$ \\
\hline \multirow[t]{4}{*}{ Trombose } & I B & Contínuo & 0 & 10 & $0,00 \%$ & \\
\hline & II D & Contínuo & 4 & 5 & $44,44 \%$ & $\mathbf{P}=\mathbf{0 , 0 3 2} *$ \\
\hline & I D & Pulsado & 0 & 10 & $0,00 \%$ & Dispensa \\
\hline & II B & Pulsado & 0 & 9 & $0,00 \%$ & análise \\
\hline \multirow[t]{4}{*}{ PMN } & I B & Contínuo & 0 & 10 & $0,00 \%$ & \\
\hline & II D & Contínuo & 1 & 8 & $5,26 \%$ & $\mathrm{P}=0,473$ \\
\hline & I D & Pulsado & 0 & 10 & $0,00 \%$ & \\
\hline & II B & Pulsado & 2 & 7 & $22,22 \%$ & $\mathrm{P}=0,210$ \\
\hline
\end{tabular}


Em certos casos observaram-se fendas pequenas com extravasamento de células sanguíneas (Figura 5) como fora descrito para outros agentes físicos.

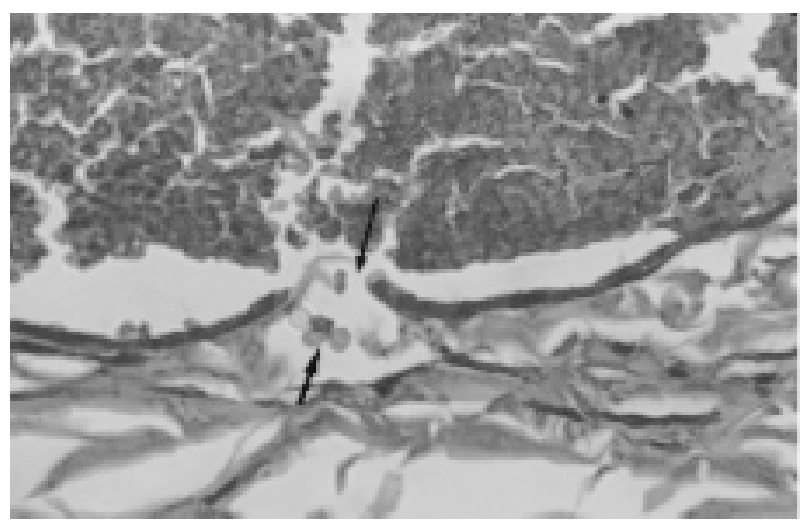

FIGURA 5- Fotomicrografia mostrando detalhe da lesão na parede vascular com extravasamento de células (setas).

\section{DISCUSSÃO}

A grande difusão do ultra-som como método terapêutico corroborou o excelente perfil de segurança observado na prática médica, paradoxalmente nem sempre baseado em estudos sistemáticos. A alteração dos níveis de potência e freqüência, assim como a preferência quase absoluta pelo ciclo pulsado - que libera a energia em períodos intermitentes - utilizados atualmente sugerem, entretanto medidas de cautela e otimização técnica, apesar de não haverem relatos de complicações com o seu emprego clínico. Encontram se poucas referências na literatura aos estudos dos bioefeitos do ultra-som no sistema circulatório ${ }^{17,18}$.

O coelho foi escolhido para o experimento devido às veias superficiais nas orelhas, de calibre e comprimento adequados ao procedimento, que garantiriam uma aplicação transcutânea sem a necessidade de dissecção. Utilizaram-se apenas animais machos para evitar a interferência do ciclo hormonal nos vasos ou gestação não identificada. Houve a perda do animal de $\mathrm{n}^{\circ} 20$ do grupo II.

Em conformidade com outros investigadores ${ }^{10,11 \text {, }}$ ${ }^{12,13}$ e por motivos de facilitação técnica, a aplicação do ultra-som foi executada com o transdutor estacionário. Este foi praticamente o único, entretanto fundamental ponto de diferenciação da utilização clínica atual.

As alterações de natureza inflamatória foram comuns às induzidas outros agentes físicos e variaram de um discreto edema até uma lesão integral da parede vascular. Algumas delas como edema, eritema, calor e até trombose, puderam ser observadas macroscopicamente.
A análise feita à microscopia óptica revelou a ocorrência da congestão e edema, também descrito por Kashyap et al $^{19}$, mas não chegou a ser estatisticamente significante, embora a utilização do ultra-som pulsado tenha promovido um aumento considerável do edema no subgrupo I D, sendo a única observação digna de nota com a utilização desta modalidade de ultra-som. Todas as outras alterações ocorridas foram com o ultrasom contínuo.

Infiltrados celulares foram observados em algumas lâminas. Sabe-se que a presença de linfócitos tem uma importante função imunológica sendo um marcador da fase crônica, provavelmente por isto só ocorreu de forma significante $(\mathrm{p}=0,032)$ no subgrupo II $\mathrm{D}$ ( 7 dias $)$, marcando o final da fase aguda. Polimorfonucleares foram identificados, mas sem significância estatística contrapondo-se aos achados descritos por Kashyap et $\mathrm{al}^{19}$. Marginalização leucocitária e presença de leucócitos na região extravascular também foram verificadas em regiões tratadas tanto com o ultra-som contínuo como no pulsado.

Trombose venosa foi o desfecho encontrado em muitos casos tratados do subgrupo II D (ultra-som contínuo), conforme descrito também por outros autores ${ }^{10,11,13}$. O mesmo tratamento, entretanto aplicado no subgrupo I B não produziu nenhum efeito identificável. A possibilidade de ser uma ocorrência mais tardia pode ter sido a causa de não ter sido verificado neste grupo cuja eutanásia foi realizada no terceiro dia. Delon-Marin et al. ${ }^{12}$ também produziram trombos na luz da veia femoral de ratos usando ultra-som focalizado de alta intensidade, porém não estavam totalmente aderidos e não verificaram a persistência do trombo no único animal do grupo examinado no $30^{\circ}$ dia do experimento. Em um outro estudo ${ }^{11}$ semelhante a este, foi aventada a possibilidade de que a elevação de temperatura pudesse ser responsabilizada pela trombose, entretanto esta variável não foi mensurada neste experimento. WILLIAMS e CHATER ${ }^{20}$ destacam que a trombose possa ocorrer não somente por lesão endotelial direta, mas também por ativação plaquetária.

Em alguns casos foi possível identificar uma completa destruição da parede vascular embora não houvesse a necrose descrita por outros investigadores ${ }^{21}$, mas com histologia semelhante ao processo natural de reabsorção vascular que ocorre após uma flebite.

Verificou-se ainda a perda dos limites da parede venosa e do endotélio com hemorragia e infiltrado inflamatório. Formação de vacúolos, degeneração e necrose das células musculares lisas na média complementaram o rol de alterações descrito por outros autores $^{10,11,22 \text {, }}$. 
Constricção, parada do fluxo, necrose de parede e hemorragia em ramos da veia femoral de coelhos devido ao efeito de cavitação do ultra-som foram descrito ${ }^{21}$ com o sistema focalizado de alta intensidade. Neste experimento, que empregou baixa intensidade, os efeitos não foram tão intensos.

Os efeitos da exposição vascular provavelmente dependem do tamanho do vaso e dos parâmetros de exposição como freqüência, duração do pulso e intensidade.

A vulnerabilidade do tipo de vaso e o grau de exposição do mesmo permitem uma grande influência no conteúdo e no continente vascular.

Corroborando com a afirmação de BARNETT et al. ${ }^{22}$ seria razoável considerar que diferentes resultados provêem desta complexa interação entre o ultra-som e os tecidos vivos, que são circunstancialmente dependentes das condições do experimento. O potencial lesivo do ultra-som aplicado externamente deve ser considerado à luz de extensa experiência com a aplicação clínica $^{24}$.

O emprego do ultra-som em doses habituais para fisioterapia, embora não apresentem riscos na prática clínica, não deve ser compreendido como uma manifestação de absoluta segurança nesta condição específica. Como visto neste trabalho, pequenas variações na freqüência, potência, tempo, forma de aplicação ou temperatura, potencialmente podem gerar efeitos diferentes mesmo com baixa potência. A prudência do seu uso deve ser considerada, sempre atentando para a correta técnica com mobilização constante do transdutor.

\section{CONCLUSÕES}

A utilização do ultra-som na potência de $3 \mathrm{~W} / \mathrm{cm}^{2}$ e $3 \mathrm{MHz}$, na forma estacionária e modo contínuo induz à trombose venosa e aumento dos linfócitos de forma significativa, o que não foi observado com o modo pulsado Considerando que o único parâmetro diferente da utilização clínica foi a forma estacionária da aplicação, recomenda-se cautela pelo risco potencial para o sistema vascular.

\section{REFERÊNCIAS}

1. American Institute Of Ultrasound In Medicine (AIUM). Bioeffects Committee. Bioeffects considerations for the safety of diagnostic ultrasound. J. Ultrasound Med 1988; 7 (9): S1S38.

2. Ter Haar GR, Daniels S. Evidence for ultrasonically induced cavitation in vivo. Phys Med Biol 1981; 26(6):1145-9.

3. Dyson M. Non-thermal cellular effects of ultrasound. Br J Cancer 1982; 45: 165-71.

4. Barnett SB, Ter Haar GR, Ziskin MC, Nyborg WL, Maeda K, Bang J. Current status of research on biophysical effects of ultrasound. Ultrasound Med Biol 1994; 20 (3): 205-18.
5. Kornowski R, Meltzer RS, Chernine A, Vered Z, Battler A. Does external ultrasound accelerate thrombolysis? results from a rabbit model. Circulation 1994; 89(1): 339-44

6. Vaezy S, Martin R, Yaziji H, Kaczkowski P, Keilman G, Carter S, Caps M, Chi EY, Bailey M, Crum L. Hemostasis of punctured blood vessels using high-intensity focused ultrasound. Ultrasound Med Biol 1998; 24(6): 903-10.

7. Vaezy S, Martin R, Kaczkowski P, Keilman G, Goldman B, Yaziji H, Carter S, Caps M, Crum L. Use of high-intensity focused ultrasound to control bleeding. J Vasc Surg 1999; 29(3): 533-42

8. Riggs PN, Francis CW, Bartos SR, Penney DP. Ultrasound enhancement of rabbit femoral artery thrombolysis. Cardiovasc Surg 1997; 5(2): 201-7.

9. Hogan RD; Franklin TD, Fry FJ, Avery KS, Burke KM. The effect of ultrasound on microvascular hemodynamics in skeletal muscle: effects on arterioles. Ultrasound Med Biol 1982; $8(1): 45-55$.

10. Fallon JT, Stehbens WE, Eggleton RC. Effect of ultrasound on arteries. Arch Pathol 1972; 94: 380-8.

11. Kerr CL, Gregory DW, Chan KK, Watmough DJ, Wheatley DN. Ultrasound-induced damage of veins in pig ears, as revealed by scanning electron microscopy. Ultrasound Med Biol 1989; 15(1):45-5.

12. Delon-Martin C, Vogt, C, Chignier E, Guers C, Chapelon JY, Cathignol, D. Venous thrombosis generation by means of highintensity focused ultrasound. Ultrasound Med Biol 1995; 21(1):113-9.

13. Walmsley AD, Laird WR, Williams AR. Intra-vascular thrombosis associated with dental ultrasound. J Oral Pathol 1987; 16(5): 256-9.

14. Hynynen K, Chung AH, Colucci, V, Jolesz FA. Potential adverse effects of high-intensity focused ultrasound exposure on blood vessels in vivo. Ultrasound Med Biol 1996; 22(2): 193-201.

15. Siegel S, Castellan JR. Nonparametrics statistics. 2ed. New York: Mc. Graw_Hill Int. ED; 1988.

16. Remington RD, Eschork MA. Estatistic with application to biological and health sciences. New Jersey: Prentice-Hall Inc; 1970 .

17. Goss SA, Johnston RL, Dunn Fry. Comprehensive compilation of empirical ultrasonic properties of mammalian tissues. J Accoust Soc Am 1978; 64(2): 423-57.

18. Stewart HD, Stewart HF, Moore RM, Jr DMV, Garry J. Compilation of reported biological effects data and ultrasound exposure levels. J Clin Ultrasound 1985; 13:167-86.

19. Kashyap A, Blinc A, Marder VJ, Penney DP, Francis CW. Acceleration of fibrinolysis by ultrasound in a rabbit ear model of small vessel injury. Thromb Res 1994; 76(5): 475-85.

20. Williams AR, Chater BV. Mammalian platelet damage in vitro by an therapeutic device. Arch Oral Biol 1980; 25: 175-9.

21. Hynynen K, Chung AH, Colucci, V, Jolesz FA. Potential adverse effects of high-intensity focused ultrasound exposure on blood vessels in vivo. Ultrasound Med Biol 1996; 22(2): 193-201.

22. Seidl M, Steinbach P, Wörle K, Hofstädter F. Induction of stress fibers and intercellular gaps in human vascular endothelium by shock waves. Ultrasonics 1994; 32(5): $397-$ 400 .

23. Barnett SB, Ter Haar GR, Ziskin MC, Nyborg WL, Maeda K, Bang J. Current status of research on biophysical effects of ultrasound. Ultrasound Med Biol 1994; 20 (3): 205-18.

24. Riggs PN, Francis CW, Bartos SR, Penney DP. Ultrasound enhancement of rabbit femoral artery thrombolysis. Cardiovasc Surg 1997; 5(2): 201-7. 
$\overline{\text { Araújo M, Baptista-Silva JCC, Gomes PO, Novo NF, Juliano Y. Effects of low intensity ultrasound }}$ in the auricular vein of rabbits. Acta Cir Bras [serial online] 2003 Jan-Feb;18(1). Available from URL: http://www.scielo.br/acb.

ABSTRACT - Purpose: The purpose of this experimental work was evaluate the effects of low intensity in the auricular vein of rabbits. Methods: Twenty rabbits were divided in two groups of ten animals. The groups differed about the place where the continuous and pulsed ultrasound were applied and the period that the material was collected for the morphologic examination ( 3 and 7 days). Acoustic coupling gel was used on marginal ear vein, each animal underwent continuous and pulsed ultrasound treatment, in segments previously marked with indelible ink. Each animal provided its own control. Ultrasound was used in the frequency of $3 \mathrm{MHz}$, intensity of $3 \mathrm{~W} / \mathrm{cm}^{2}$ in the pulsed and continuous modes during $10 \mathrm{~min}$ in a stationary way. The group I was euthanized after 3 days and the group II after 7 days contemplating the acute phase of the inflammatory process. We applied the Fisher and Mc Nemar's tests for statistical analysis. Results: Venous thrombosis and lymphocytes increment occurred significantly $(p=0,032)$ due continuous mode ultrasound. Other minor findings were congestion, edema, rupture of vessel wall and hemorrhage. Conclusions: continuous ultrasound causes venous thrombosis and lymphocytes increment.

KEY WORDS - Ultrasound. Ultrasound therapy. Vascular. Veins. Rabbit.

Conflito de interesse: nenhum

Correspondência:

Fonte de financiamento: nenhuma

Marcelo Araújo

Rua Rui Barbosa, 376/801

45600-901 Itabuna - BA

Tel: (73) 212-2662

Fax: (73) 617-0011

marcelo_araujo_ahotmail.com

Data do recebimento: 25/09/2002

Data da revisão: 14/10/2002

Data da aprovação: 29/10/2002

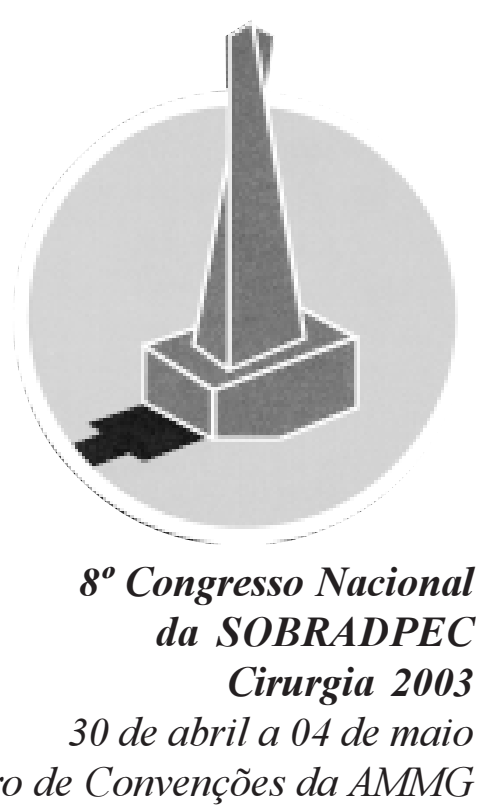

